\title{
Modélisation dynamique des systèmes électriques insulaires incluant des énergies renouvelables : hydraulique et éolienne
}

\author{
Dynamic modelling of autonomous power systems including renewable \\ power sources
}

par G. N. Kariniotakis

Ecole des Mines de Paris, Centre d'Energétique

The use of renewable energies for electricity production presents a growing interest, especially in autonomous power systems where the cost of conventional production is high. In recent years, the integration of wind energy is seen as an attractive alternative for fuel displacement. However, the intermittent nature of wind production imposes several difficulties to the power system operation when penetration is high. Here, a model is developed for the transient stability analysis of isolated power systems including various conventional (thermal, gas, diesel) and renewable (hydro, wind) power units. The objective is to assess the impact of a high integration from the renewables and to focus on the interaction between the hydro units - that have a special dynamic behaviour -, and the wind turbines. Detailed models for each one of the power system components are developed. Emphasis is given in the representation of different hydro power plant structures. Finally, the casestudy of the power system of New Caledonia is examined, where various types of hydro plants and a wind farm are installed. The developed model permits to define the penetration limits of the renewable sources, to assess the system stability and finally it contributes in the dimensioning of new power plant installations.

\section{IINTRODUCTION}

L'intégration des énergies renouvelables (EnR) - hydraulique et éolienne - dans les systèmes insulaires, où le coût de la production conventionnelle est élevé, présente un grand intérêt grâce aux économies de combustible réalisées et aux aspects environnementaux. En dépit de leurs nombreux avantages, l'inconvénient majeur des EnR, et particulièrement de l'énergie éolienne, reste leur nature aléatoire. A cause de cette particularité, leur intégration dans un système insulaire est limitée par des contraintes techniques. La recherche d'un compromis entre fonctionnement économique (maximisation de l'intégration des EnR) et sécurité du système électrique, rend la gestion du système électrique plus complexe pour les opérateurs [4].

Le modèle dynamique présenté ici permet d'analyser la sécurité du système électrique par rapport aux fluctuations des ressources renouvelables. Il permet d'évaluer la stabilité du système (variations de fréquence et de tension) suite à des perturbations comme des connexions/déconnexions des machines, de courts-circuits, de rafales de vent etc., dans une échelle de temps entre 0 et $30 \mathrm{sec}$. Le modèle permet d'établir des règles de fonctionnement du système électrique dans les normes acceptables, en définissant des limites supérieures de pénétration des EnR. De plus, il permet de désigner et de placer des moyens de protection pour l'amélioration de la sécurité du réseau. Finalement, le modèle dynamique contribue au problème de dimensionnement d'une nouvelle installation EnR par la définition du niveau supérieur de l'intégration des EnR. Le modèle dynamique contient des modèles pour $[1-3,5]$ :

- les unités thermiques,

- les turbines à gaz,

- les groupes diesel,

- les turbines hydrauliques,

- les éoliennes,

- des types de charges différents.

Selon le type d'unité, des modèles appropriés pour les génératrices synchrones, asynchrones, les régulateurs de 
vitesse et de tension, ont été considérés. Une attention particulière a été portée sur la modélisation détaillée des centrales hydrauliques afin de représenter avec précision leur comportement dynamique ainsi que l'impact dû au couplage avec d'autres sources intermittentes comme les éoliennes. Un modèle détaillé a donc été développé pour chaque type de centrale hydraulique en prenant en compte sa propre configuration structurelle (chemin d'équilibre, conduites,...).

Le modèle développé permet la simulation des perturbations comme des courts-circuits, de perte de charge, de connexions ou de déconnexions des groupes électriques, de production variable des sources renouvelables, la perte d'une ferme éolienne etc.

Le cas d'étude de la Nouvelle Calédonie est considéré. La particularité de cette île est qu'un grand pourcentage (de l'ordre de $30 \%$ ) des besoins énergétiques est couvert par des énergies renouvelables. La plus grande partie concerne l'énergie hydraulique. Il existe aussi une ferme éolienne de 2,7 MW composée de 12 machines de $225 \mathrm{~kW}$. Une caractéristique de ce système est la forte demande industrielle qui représente $70 \%$ de la demande totale. Un modèle du réseau de l'île est développé et les résultats de l'analyse du régime stable sont comparés aux mesures obtenues par le système d'acquisition de données de l'île.

Afin d'examiner l'impact d'une intégration éolienne plus importante (jusqu'à $25 \%$ ) sur la stabilité du système électrique, des scénarios avec des fermes éoliennes supplémentaires de $20 \mathrm{MW}$ ont été considérés.

\section{MODÉLISATION D'UN SYSTÈME ÉLECTRIQUE INSULAIRE INCLUANT DES ÉNERGIES RENOUVELABLES}

Dans cet article, le modèle complet d'un groupe hydraulique, comprenant des équations pour la turbine hydraulique, la génératrice synchrone et les régulateurs de vitesse et de tension est présenté. Les équations décrivant le comportement dynamique d'une éolienne sont ainsi données. Les modèles pour les groupes diesel sont décrits en [1], alors que les modèles des groupes thermiques le sont en [5].

\subsection{Le modèle de la génératrice synchrone}

Le modèle de la génératrice synchrone est du $5^{\mathrm{c}}$ ordre et comprend les équations du mouvement du rotor, les phénomènes dynamiques liés à l'enroulement de l'excitation de l'alternateur, ainsi que deux enroulements amortisseurs rotoriques, l'un dans l'axe direct et l'autre dans l'axe en quadrature. Les transitoires du stator correspondant aux termes de dérivées du flux, connus sous le nom de f.é.m. de transformation, sont négligés étant plus rapides que les transitoires du rotor. Cette simplification permet d'obtenir des équations algébriques pour le stator. L'angle rotorique $\delta$ de l'alternateur est :

$$
\frac{d \delta}{d t}=\omega-\omega_{0}=\Delta \omega
$$

où $\omega$ est la vitesse de rotation et $\omega_{0}$ est la vitesse de synchronisme en radians électriques par seconde. L'accélération du rotor est donnée par :

$$
\frac{d \omega}{d t}=\frac{\omega_{0}}{2 H}\left(T_{m}-T_{e}-\frac{D_{A}}{\omega_{0}} \Delta \omega\right)
$$

où $H$ est la constante d'inertie du groupe, $T_{m}$ est le couple mécanique net fourni par la turbine (en p.u. $\frac{m}{-}$ «per unit ») et $T_{e}$ est le couple électromagnétique développé par l'alternateur (en p.u.) :

$$
T_{e}=E_{d}^{\prime \prime} I_{d}+E_{q}^{\prime \prime} I_{q}-\left(X_{d}^{* \prime}-X_{q}^{\prime \prime}\right) I_{d} I_{q}
$$

Les équations de l'enroulement d'excitation et des deux enroulements amortisseurs du rotor sont en p.u :

$$
\begin{aligned}
& \frac{d E_{q}^{\prime}}{d t}=\frac{1}{T_{d o}^{\prime}}\left(E_{f d}-\frac{X_{d}-X_{d}^{*}}{X_{d}^{\prime}-X_{d}^{\prime \prime}} E_{q}^{\prime}+\frac{X_{d}-X_{d}^{\prime}}{X_{d}^{\prime}-X_{d}^{\prime \prime}} E_{q}^{\prime}\right) \\
& \frac{d E_{d}^{\prime}}{d t}=-\frac{1}{T_{q o}^{\prime}}\left[E_{d}^{\prime \prime}-\left(X_{q}-X_{q}^{\prime \prime}\right) I_{q}\right] \\
& \frac{d E_{q}^{\prime \prime}}{d t}=-\frac{1}{T_{d o}^{*}}\left[E_{q}^{\prime \prime}-E_{q}^{\prime}+\left(X_{d}^{\prime}-X_{d}^{\prime \prime}\right) I_{d}\right]
\end{aligned}
$$

Les équations algébriques du stator sont en p.u. :

$$
V_{d}=E_{d}^{*}-r_{s} I_{d}+X_{q}^{*} I_{q}, \quad V_{q}=E_{q}^{*}-r_{s} I_{q}-X_{d}^{*} I_{d}
$$

La tension de sortie de la machine, le courant injecté au réseau ainsi que les puissances active et réactive fournie par la machine sont donnés (en p.u.) par :

$$
\begin{gathered}
V_{t}=\sqrt{V_{d}^{2}+V_{q}^{2}}, I_{A}=\sqrt{I_{d}^{2}+I_{q}^{2}} \\
P_{A e}=\operatorname{Re}\left\{V_{t} I_{A}^{*}\right\}, Q_{A e}=\operatorname{Im}\left\{V_{t} I_{A}^{*}\right\}
\end{gathered}
$$

\subsection{Régulateur automatique de tension}

Le programme de simulation développé a une structure modulaire, de sorte que différents types de régulations primaires peuvent être utilisés pour chaque groupe. Deux modèles typiques pour le régulateur de tension sont les systèmes de type 1 et 2 de l'IEEE décrits par les équations suivantes:

Régulateur :

$$
\frac{d V_{R}}{d t}=\frac{1}{T_{R}}\left(K_{R} V_{t}-V_{R}\right)
$$

Amplificateur :

$$
\frac{d V_{A}}{d t}=\frac{1}{T_{A}}\left(K_{A} V_{e}-V_{A}\right)=\frac{1}{T_{A}}\left[K_{A}\left(V_{r e f}+\frac{V_{s}}{K_{A}}-V_{R}-V_{F}\right)-V_{A}\right]
$$

avec

$$
V_{R \min } \leq V_{A} \leq V_{R \max }
$$

Excitatrice :

$$
\frac{d V_{r}}{d t}=\frac{1}{T_{E}}\left(V_{A}-V_{E}-K_{E} V_{r}\right)
$$

Stabilisateur :

$$
\begin{aligned}
& \frac{d V_{F}}{d t}=\frac{1}{T_{F}}\left(K_{F} \frac{d V_{F F}}{d t}-V_{F}\right)= \\
& =\left\{\begin{array}{l}
\frac{1}{T_{F}}\left[\frac{K_{F}}{T_{E}}\left(V_{A}-V_{E}-K_{E} V_{r}\right)-V_{F}\right] \quad \text { pour Type 1 }\left(V_{F F}=V_{r}\right) \\
\frac{1}{T_{F}}\left[\frac{K_{F} K_{A}}{T_{A}}\left(V_{r e f}+\frac{V_{s}}{K_{A}}-V_{R}\right)\right.
\end{array}\right. \\
& \left.-\frac{K_{F}}{T_{A}} V_{A}-\left(1+\frac{K_{E} K_{F}}{T_{A}}\right) V_{F}\right] \text { pour Type } 2\left(V_{F F}=V_{A}\right)
\end{aligned}
$$


L'interface entre le régulateur de tension et la génératrice synchrone est donné par :

$$
E_{f d}=K_{f} V_{r}
$$

\subsection{La turbine hydraulique}

Dans cette étude on s'intéresse aux types d'installations hydrauliques :

- d'une turbine alimentée par une conduite avec ou sans cheminée d'équilibre,

- de $n$ turbines alimentées par le même tunnel avec ou sans cheminée d'équilibre.

Pour chaque turbine hydraulique, indépendamment du type d'installation, une génératrice synchrone et un régulateur de tension sont considérés, décrits par les modèles des paragraphes précédents. L'objectif de cette section est de donner les équations qui permettent d'obtenir le couple mécanique développé par la turbine et nécessaire dans l'équation d'accélération de la génératrice.

\subsubsection{Modélisation des phénomènes transitoires d'une turbine hydraulique}

Le cas le plus simple d'une turbine est considéré avec une conduite et sans cheminée d'équilibre. L'eau est supposée se comporter en fluide incompressible. La dynamique du débit $q$ dans la conduite, qui est d'une longueur $L$ et d'une section $A$, est donnée en p.u. par :

$$
\frac{d q}{d t}=\frac{1}{T_{w}}\left(1-h-h_{t}\right)
$$

où $h$ est la pression en amont de la turbine, $h_{l}$ représente la perte de charge due aux frottements dans la conduite et $T_{w}$ est le temps de lancer de la conduite (en sec) défini comme:

$$
T_{w}=\frac{L}{A g} \frac{Q_{\text {base }}}{H_{\text {base }}} \sec
$$

où $Q_{\text {base }}$ et $H_{\text {base }}$ sont le débit et la pression statique et $g$ est l'accélération de la pesanteur $\left(9,81 \mathrm{~m} / \mathrm{s}^{2}\right)$. La turbine est représentée de manière simple, par la caractéristique d'une vanne : $q=G \sqrt{h}$, où $G$ est la position du cercle de vannage en p.u. La puissance mécanique développée par la turbine est en p.u. :

$$
P_{m}=A_{t} h\left(q-q_{n l}\right)-D G \Delta \omega
$$

où $q_{n l}$ est le débit (en p.u.) de fonctionnement de la turbine sans charge et $A_{t}$ est une constante. En négligeant les pertes, et après linéarisation, la fonction de transfert suivante est obtenue où $G_{0}$ est le débit au régime stable :

$$
\frac{\Delta P_{m}}{\Delta G}=\frac{1-G_{0} T_{w} s}{1+\frac{G_{0} T_{w} s}{2}} \cdot A_{t}
$$

La figure $l$ montre la variation (\%) de la puissance mécanique d'une turbine suite à une ouverture de 0,2 p.u. de la position de la vanne. Pour des raisons de comparaison, l'action d'un régulateur de vitesse n'est pas prise en compte dans cet exemple où $T_{w}=1,83 \mathrm{~s}, A_{t}=1,004$ et $G_{0}=0,762$ p.u. Malgré l'augmentation de la charge de la turbine due à l'ouverture de la vanne, la puissance mécanique est réduite instantanément de $60 \%$. Le modèle linéaire est plus pessimiste. Si l'action du régulateur de vitesse est prise en compte, la chute observée est partiellement amortie. Cet exemple présente clairement la particularité du comportement transitoire d'une turbine hydraulique.

\subsubsection{Régulateur de vitesse d'une turbine hydraulique.}

La figure 2 montre le schéma global des fonctions de transfert d'un régulateur de vitesse typique pour une turbine hydraulique. Le schéma correspond à 4 équations différentielles qui sont résolues en parallèle avec les équations de mouvement, ainsi que celles de la génératrice synchrone, du régulateur de tension et de la turbine hydraulique.

\subsubsection{Simulation des effets de la cheminée d'équi- \\ libre.}

Dans certains cas où la centrale hydraulique est éloignée du réservoir, une cheminée d'équilibre près de la turbine est nécessaire afin d'amortir les phénomènes dynamiques liés aux variations du débit. Le cas le plus simple d'un tel système est donné en figure 3. La réponse transitoire de la turbine est simulée suite à une augmentation de 0,1 p.u. de sa charge. L'action du régulateur de vitesse est prise en compte. La cheminée d'équilibre n'affecte pas de façon significative le comportement transitoire, à très court terme. Son influence apparaît dans des périodes plus longues qui sont comparables à sa fréquence propre. Une comparaison avec un modèle qui néglige la cheminée d'équilibre met en évidence l'effet long terme de cet élément.
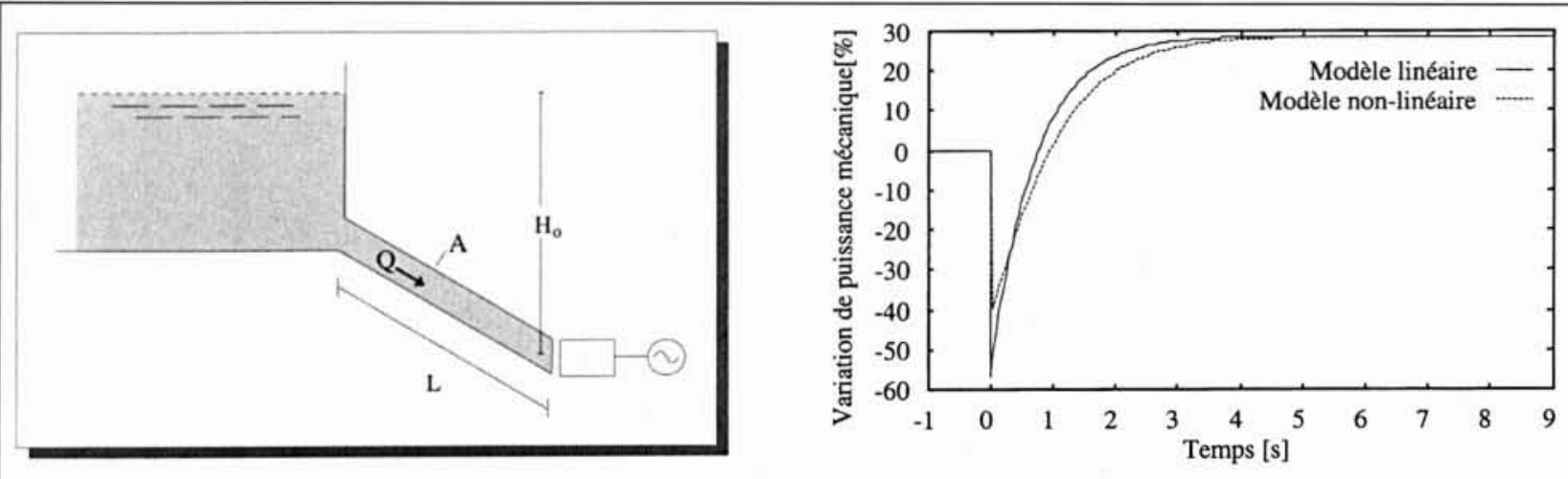

1. Variation $(\%)$ de la puissance mécanique suite à une ouverture de 0,2 p.u. de la position de vanne. Comparaison des réponses obtenues par le modèle linéaire (15) et non-linéaire (12). 


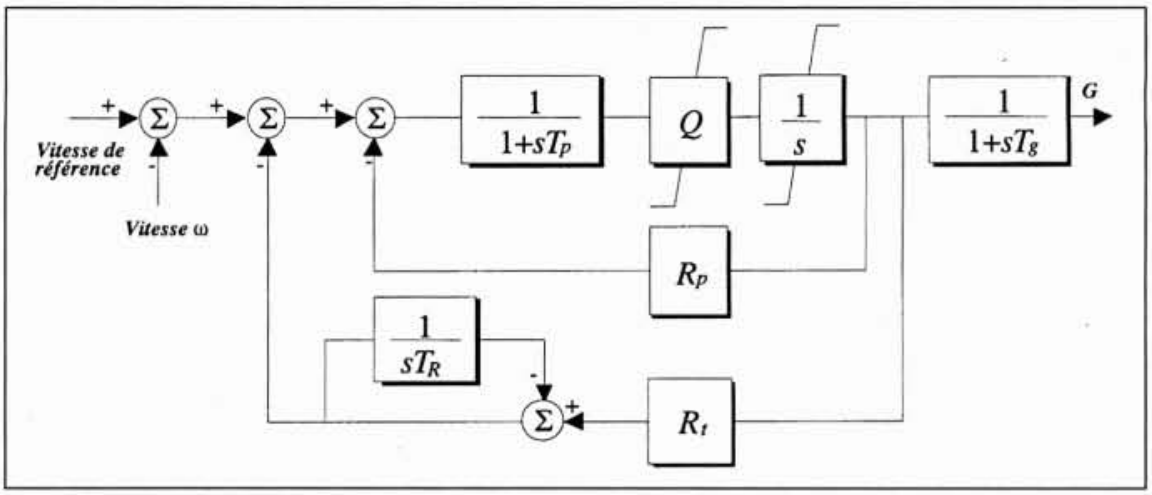

2. Régulateur de vitesse d'une turbine hydraulique.

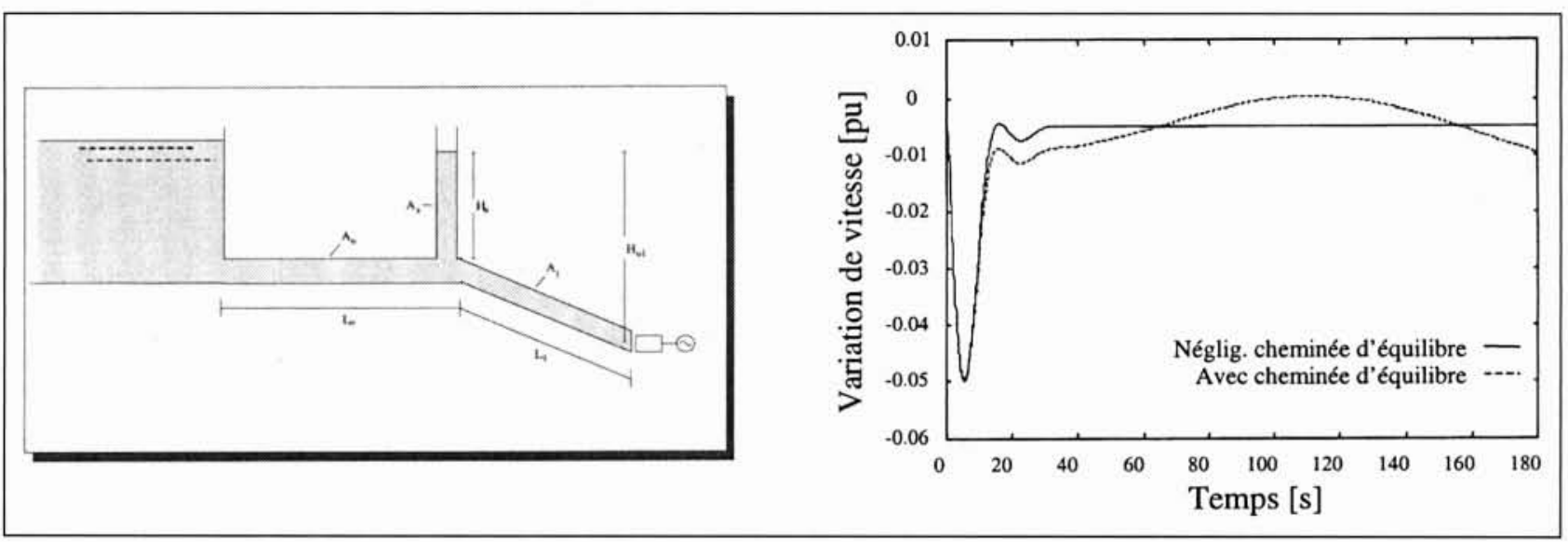

3. (Gauche) Installation hydraulique avec cheminée d'équilibre. (Droite) Influence de la cheminée d'équilibre au comportement dynamique de la turbine. Comparaison entre un modèle qui prend en compte cet élément pour une période de 3 minutes et un autre qui le néglige. Variation de vitesse de la turbine suite à une augmentation de 0,1 de la charge.

3.2.4 Simulation d'un système de turbines alimenté par un tunnel commun.

Dans certains systèmes, un nombre de turbines hydrauliques est alimenté par un tronçon de conduite commune (fig. 4,7). Dans ce cas il existe une interaction hydraulique entre les groupes. La figure 4 montre la réponse dynamique d'un tel système de deux turbines suite à une ouverture de 0,1 p.u. de la vanne de la deuxième turbine. A cause de ce changement, on observe une variation de $20 \%$ de la puissance mécanique de la deuxième turbine. Cette variation est due aux conduites communes des deux turbines. La dynamique est décrite par l'équation suivante (en p.u.):

$$
\left[\begin{array}{l}
\dot{q}_{1} \\
\dot{q_{2}}
\end{array}\right]=\left[\begin{array}{cc}
T_{w}+T_{w 1} & T_{w} \\
T_{w} & T_{w}+T_{w 2}
\end{array}\right]^{-1} \cdot\left[\begin{array}{c}
1-h_{1} \\
1-h_{2}
\end{array}\right]
$$

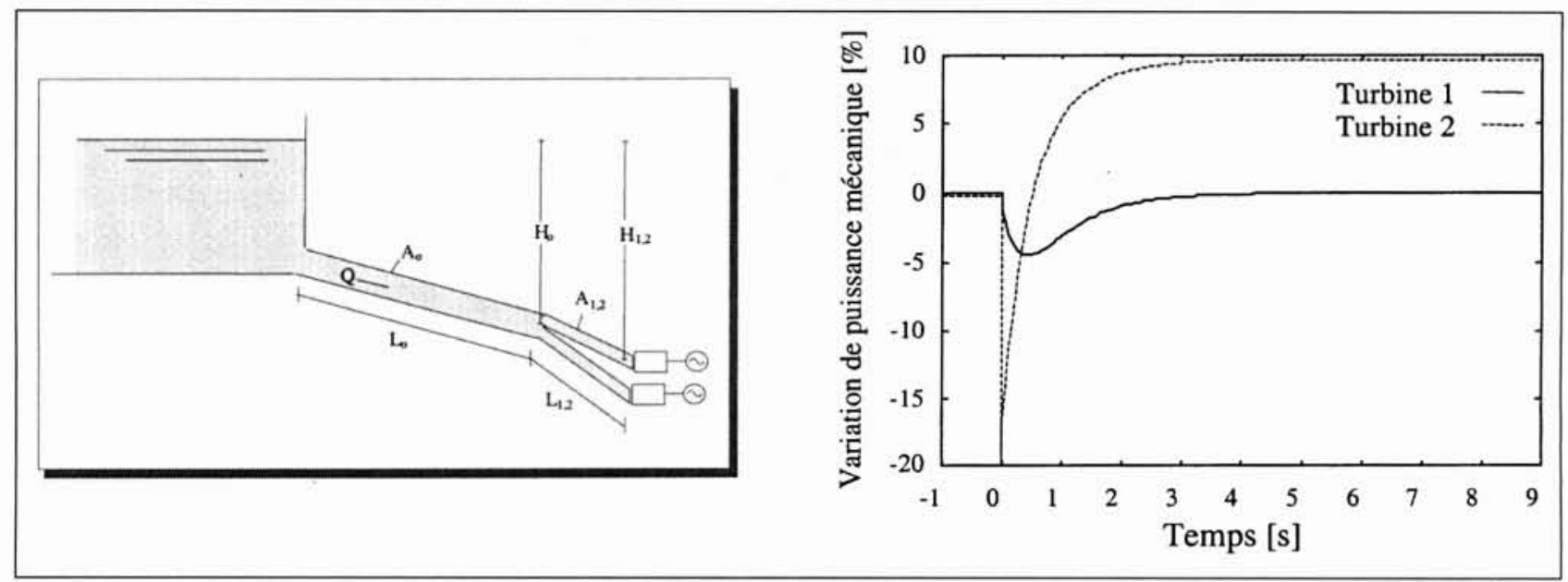

4. (Gauche) Installation hydraulique avec deux turbines alimentées par un tunnel commun. (Droite) Réponse de la puissance mécanique de deux turbines suite à une ouverture de 0,1 p.u de la vanne de la deuxième turbine. 
où $T_{w}$ est le temps de lancer du tunnel et $T_{w 1}, T_{w 2}$ sont les constantes de temps de lancer des deux conduites définies d'une façon similaire comme en (14). A chaque turbine des équations différentes concernant la génératrice synchrone et les régulateurs de vitesse et de tension sont associées.

\subsection{Le modèle d'un générateur éolien}

La puissance mécanique développée au rotor d'une turbine éolienne est donnée par:

$$
P_{A m}=\left\{\begin{array}{cl}
0 & \text { si } U_{w}<U_{c i} \text { ou } U_{w}>U_{c o} \\
0,5 \rho C_{p} A U_{w}^{3} & \text { si } U_{c i} \leq U_{w} \leq U_{r} \\
P_{n} & \text { si } U_{r} \leq U_{w} \leq U_{c o}
\end{array}\right.
$$

où $U_{w}$ est la vitesse instantanée du vent, $r$ est la masse volumique de l'air, $A$ est la surface balayée par l'hélice et $C_{p}$ est le facteur de conversion de la puissance cinétique du vent en puissance mécanique [1]. L'accélération du rotor est donnée par l'équation du mouvement :

$$
\frac{d \omega_{A}}{d t}=\frac{\omega_{0}}{2 H_{A}}\left(T_{A m}-T_{A e}-\frac{D_{A}}{\omega_{0}} \omega_{A}\right)
$$

où $H_{A}$ est l'inertie de la machine et $\omega_{A}$ est la vitesse de rotation en $\mathrm{rad} / \mathrm{sec}$. Le cas d'une éolienne équipée d'une génératrice asynchrone est considéré. Le modèle d'un moteur asynchrone en mode de fonctionnement de générateur (« charge négative ») est donc utilisé. Les équations différentielles décrivant la dynamique des enroulements du rotor sont en p.u. :

$$
\begin{aligned}
& \frac{d E_{d}^{\prime}}{d t}=\frac{1}{T_{0}^{\prime}}\left[-E_{d}^{\prime}-\left(X-X^{\prime}\right) I_{q}\right]+s \omega_{e} E_{q}^{\prime} \\
& \frac{d E_{q}^{\prime}}{d t}=\frac{1}{T_{0}^{\prime}}\left[-E_{q}^{\prime}+\left(X-X^{\prime}\right) I_{d}\right]-s \omega_{e} E_{d}^{\prime}
\end{aligned}
$$

où $s$ est le glissement défini comme $s=\left(\omega_{e}-\omega_{A}\right) / \omega_{e}$. Comme pour la machine synchrone, les transitoires du stator sont négligés étant plus rapides que les transitoires du rotor. Les équations algébriques suivantes sont obtenues en p.u. :

$$
V_{d}=E_{d}^{\prime}+r_{s} I_{d}-X^{\prime} I_{q}, \quad V_{q}=E_{q}^{\prime}+r_{s q} I_{q}+X^{\prime} I_{d}
$$

où $r_{s}$ est la résistance du stator. Le couple électromagnétique développé par l'alternateur est :

$$
T_{A e}=E_{d} I_{d}+E_{q}^{\prime} I_{q}
$$

La tension, le courant injecté au réseau ainsi que la puissance active et réactive fournie par la machine sont donnés par les mêmes équations comme pour la machine synchrone.

Dans le cas d'une ferme éolienne comprenant plusieurs machines, le nombre des équations différentielles devient important. Lorsqu'un nombre $n$ de machines identiques fonctionne sous les mêmes conditions de vent, il est possible de considérer dans la simulation une machine équivalente qui remplace les $\mathrm{n}$ machines. Cette approche permet la réduction du nombre totale des équations. Les paramètres de la machine équivalente sont donnés par:

$$
H_{A, e q}=n H_{A}, T_{0, e q}^{\prime}=T_{0}^{\prime}, r_{s, e q}=\frac{r_{s}}{n}, X_{e q}=\frac{X}{n}, X_{e q}^{\prime}=\frac{X}{n}
$$

\subsection{Le réseau électrique}

Le réseau de transport interconnectant des machines électriques est modélisé en utilisant la représentation détaillée de ses divers éléments. Cela inclut la représentation par équivalent en $\pi$ des lignes de transmission (résistance, réactance, capacité), les transformateurs simples ou exploités sur une prise ne correspondant pas à leur rapport nominal (transformateurs pour la régulation du niveau et de la phase de tension), les compensations shunt, ainsi que les charges à impédance constante et puissance constante.

Pour un système électrique avec $N$ jeux de barres où il y a des machines synchrones et asynchrones connectées (courant injecté), le vecteur de tension $V_{N}$ des jeux de barres et les courants injectés $I_{N}$ sont reliés par la matrice d'impédances du réseau $Z_{N}$ par l'équation $V_{N}=Z_{N} I_{N}$. Cette équation est combinée avec les équations du stator des génératrices synchrones et des machines asynchrones (génératrices ou moteurs) et l'équation suivante est obtenue en forme matricielle :

$$
I_{d q}=\left[T^{-1} Z_{N}^{\prime} T+Z_{m}^{\prime}\right]^{-1} E_{d q}
$$

où $T$ est une matrice de transformation qui permet de passer du système de référence synchrone au système de référence individuel de chaque machine en fonction de l'angle $\delta$ de la machine. Les matrices $Z_{n}$ et $Z_{m}$ incluent respectivement les impédances du réseau, et celles des alternateurs. Cette équation est résolue à chaque pas d'intégration afin de donner le courant de chaque machine dans son propre système de référence.

\section{LE CAS DE LA NOUVELLE CALÉ- DONIE}

L'île de la Nouvelle Calédonie est située à $1500 \mathrm{~km}$ à l'Est de l'Australie dans l'océan Pacifique. Sa superficie est de $18760 \mathrm{~km}^{2}$ pour une longueur de $400 \mathrm{~km}$ et une largeur comprise entre 40 et $50 \mathrm{~km}$. Sa population est de 145000 habitants dont 90000 pour la capitale Nouméa. L'île dispose de plus de $25 \%$ des ressources connues de nickel au niveau mondial et elle est le $3^{\mathrm{e}}$ producteur au monde. Le nickel est donc la base principale de l'économie de l'île et cette situation influe sur sa situation énergétique.

\subsection{Description du système électrique de la nouvelle Calédonie.}

La puissance totale installée en Nouvelle Calédonie est de $280 \mathrm{MW}$. La production annuelle est de $1400 \mathrm{GWh}$ (1994, $+9 \%$ ). Le tableau 1 donne une description générale des centrales de chaque île du complexe Calédonien. Sur les îles il existe aussi un nombre de mini-réseaux isolés alimentés par des mini-centrales hydrauliques de puissance nominale entre 26 et $147 \mathrm{~kW}$ couplées à des groupes diesel. De plus, 1000 kits photovoltaïques sont installés pour l'électrification de maisons isolées.

La demande électrique de la Nouvelle Calédonie est entre 110 et $210 \mathrm{MW}$. $70 \%$ de cette demande vient du secteur industriel (pointe $126 \mathrm{MW}$ ). La demande en pointe du secteur public est de 79,5 MW (1997). $75 \%$ de la demande publique provient de la capitale Nouméa au sud de l'île. En figure 6, une journée typique est représentée (les mesures sont obtenues par le système d'acquisition de données SCADA - de l'île).

La demande industrielle de l'île vient de l'usine SLN de Nickel à Doniambo où il existe trois fours de $40 \mathrm{MW}$ chacun pour fondre le minerai. Cette demande est planifiée et reste généralement constante sur une base hebdomadaire. Des variations éventuelles sont déterminantes pour la courbe de la demande journalière comme il est montré en figure 6 . La même figure montre la répartition des moyens de produc- 


\section{Applications industrielles de la mécanique des fluides}

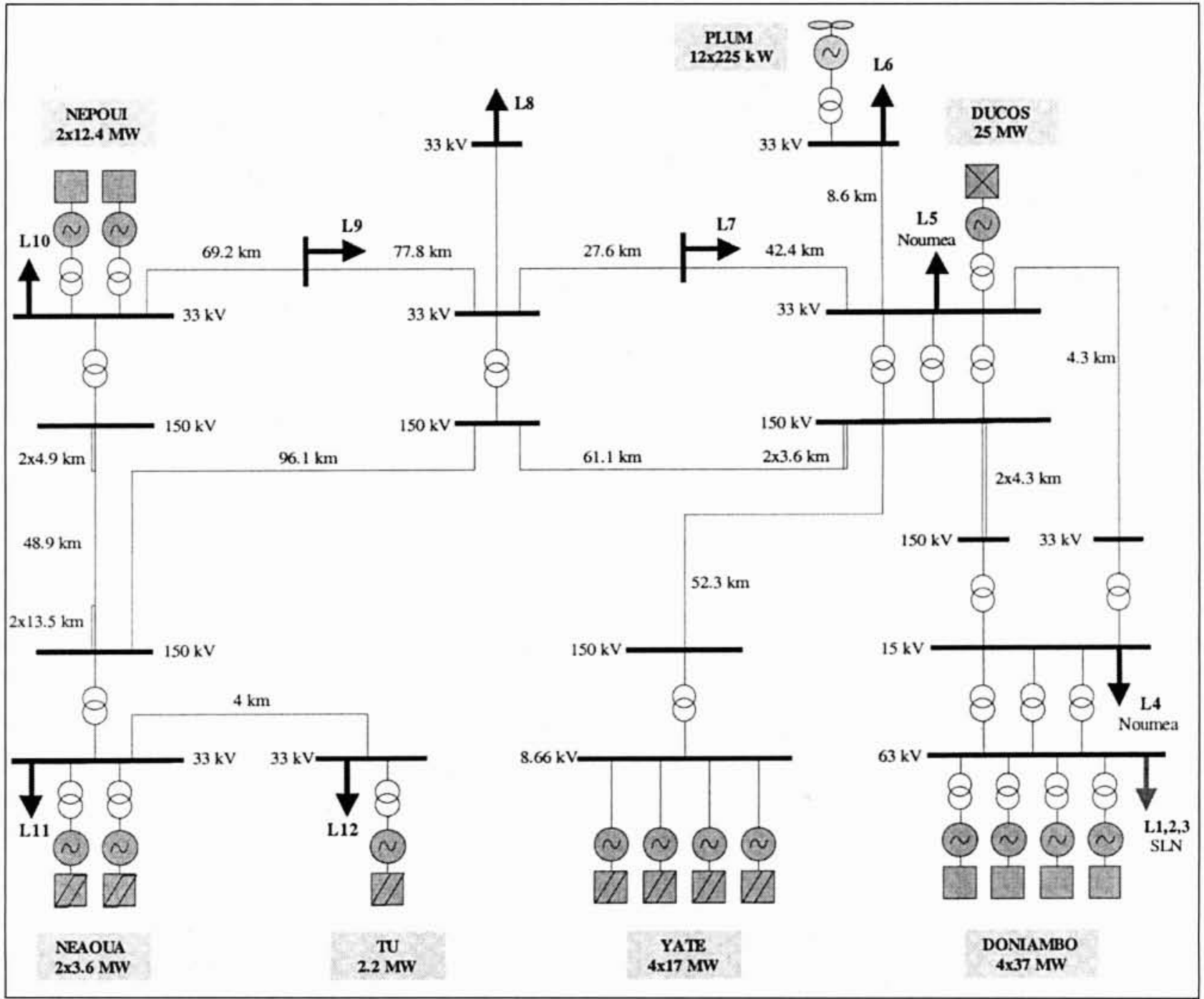

5. Schéma du système électrique de la Nouvelle Calédonie.

Centrales hydrauliques : Yaté $(4 \times 17 \mathrm{MW})$, Néaoua $(2 \times 3,6 \mathrm{MW})$ et Tu $(2,2 \mathrm{MW})$.

Centrale thermique : Doniambo $(4 \times 40 \mathrm{MW})$.

Centrale diesel : Népoui $(2 \times 12,4 \mathrm{MW})$.

Ferme éolienne: Plum $(12 \times 225 \mathrm{~kW})$.

Turbine à gaz: Ducos $(20 \mathrm{MW})$.

Tableau 1. - Puissance installée dans le complexe de la Nouvelle Calédonie.

\begin{tabular}{|c|c|c|c|c|c|c|c|c|}
\hline \multirow[b]{3}{*}{ Type de production } & \multicolumn{2}{|c|}{ Grande-Terre } & \multirow{3}{*}{$\begin{array}{l}\text { Ouvea } \\
\text { (kW) }\end{array}$} & \multirow{3}{*}{$\begin{array}{l}\text { Lifou } \\
\text { (kW) }\end{array}$} & \multirow{3}{*}{$\begin{array}{l}\text { Mare } \\
(\mathrm{kW})\end{array}$} & \multirow{3}{*}{$\begin{array}{c}\text { Ile des Pins } \\
\text { (kW) }\end{array}$} & \multirow{3}{*}{$\begin{array}{c}\text { Autres iles } \\
(\mathrm{kW})\end{array}$} & \multirow{3}{*}{$\begin{array}{l}\text { Total } \\
\text { (MW) }\end{array}$} \\
\hline & Interconnect. & Autonomes & & & & & & \\
\hline & (MW) & (MW) & & & & & & \\
\hline Thermique & 160 & & & & & & & 160 \\
\hline Hydraulique & 77,4 & 0,563 & & & & & & 77,9 \\
\hline Diesel & 24,8 & 1,68 & 1620 & 3070 & 2080 & 2140 & 570 & 35,9 \\
\hline Turbine à gaz & 20 & & & & & & & 20 \\
\hline Eoliennes & 2,7 & & & $3 \times 100$ & & $1 \times 10$ & & 3 \\
\hline Total & 284,9 & 2,243 & 1620 & 3370 & 2080 & 2150 & 296,8 & \\
\hline Demande & $110-210$ & & $150-600$ & $600-1400$ & $350-750$ & $150-350$ & & \\
\hline
\end{tabular}


tion pour couvrir la demande. Le cas de la Nouvelle Calédonie présente la particularité des contraintes contractuelles entre les organismes responsables pour la production, la distribution et aussi le secteur industriel. Ces contraintes sont représentées par la répartition des moyens de production, i.e. la centrale diesel fonctionne en base en permanence.

La compagnie locale d'électricité Enercal utilise des moyens avancés pour la gestion du système et plus précisément pour la gestion annuelle de la centrales de Yaté (hydraulique) et de Doniambo (thermique). Ces deux centrales fonctionnent alternativement en base selon la saison. Des aspects concernant les coûts de production, les probabilités d'hydraulicité, la fiabilité des groupes, les prédictions de la demande électrique etc., sont pris en compte par le logiciel de gestion.

La gestion de la centrale de Néaoua est faite sur une base hebdomadaire. Des contraintes d'alimentation en eau pour la région correspondante sont prises en compte. La plus petite centrale hydraulique de Tu est entièrement automatisée.

La demande est principalement concentrée au sud de l'île. Une réactance de 5 MVAr est installée au niveau de la centrale de Népoui pour l'absorption de la puissance réactive générée par les lignes de transmission de haute tension $(150 \mathrm{kV})$. La turbine à gaz est mise rarement en service pour couvrir les pointes. Le facteur principal pour les variations de la demande publique est la température. Les opérateurs estiment qu'une variation de $1^{\circ} \mathrm{C}$ amène à une variation de $2 \mathrm{MW}$. Cette estimation est utile pour prévoir la demande du jour suivant.

\subsection{Le comportement dynamique du système électrique}

Les variations court terme de la charge industrielle ont un impact important sur la fréquence du réseau. Par exemple des variations de $2 \mathrm{MW}$ peuvent avoir lieu en quelques secondes. Les trois fours disposent de protections pour leur déconnexion lorsque le niveau de la fréquence du réseau dépasse le seuil de $48,6 \mathrm{~Hz}$. La figure 8 montre l'évolution de la fréquence du réseau pendant un jour typique. La variation maximale correspond à une perturbation importante. Il s'agit d'une déconnexion d'un groupe thermique de $37 \mathrm{MW}$. Cette déconnexion a provoqué une chute de fréquence qui a activé les protections des fours. L'ensemble de trois fours (75 MW) a été déconnecté simultanément provoquant une variation de fréquence de $51,1 \mathrm{~Hz}$. On estime que la déconnexion d'autres éléments, i.e. de la ferme éolienne, a eu lieu par l'activation de leur protections à cause de ces variations de fréquence.

Dans le cadre d'analyses de scénarios d'une intégration éolienne plus importante, un nombre de machines supplémentaires est considéré sur le site de Plum pour attendre une puissance totale éolienne de $20 \mathrm{MW}$. La figure 9-a montre la variation de la fréquence du réseau suite à une déconnexion simultanée des machines lorsqu'elles produisent leur puissance nominale $(20 \mathrm{MW})$. La figure $9 b$ montre les variations de la tension au niveau des groupes électriques. La déconnexion provoque une augmentation de la puissance active des groupes conventionnels et une diminution de la puissance réactive. Une telle perturbation est possible suite à un
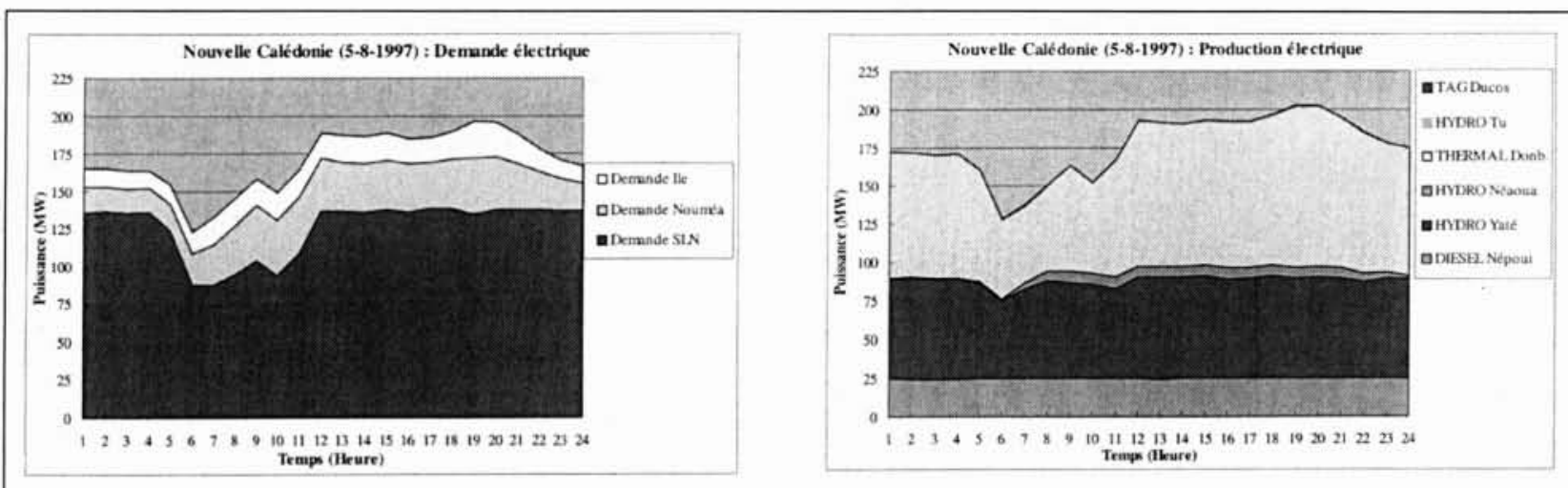

6. Profil de la demande électrique d'un jour typique en Nouvelle Calédonie.

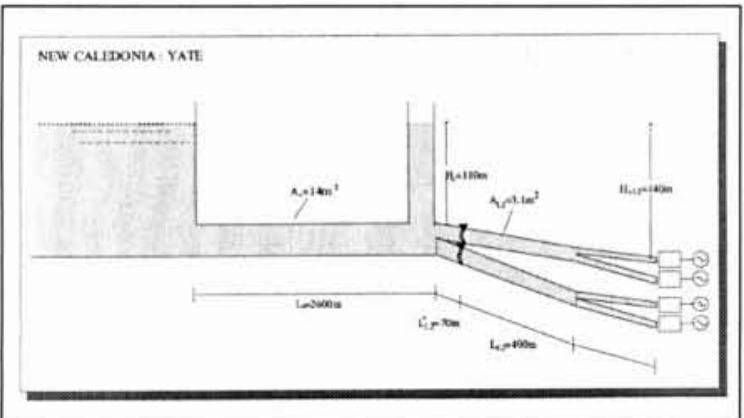

7. Structure de la centrale hydraulique de Yaté : 4 turbines de $17 \mathrm{MW}$ alimentées par un tunnel commun.

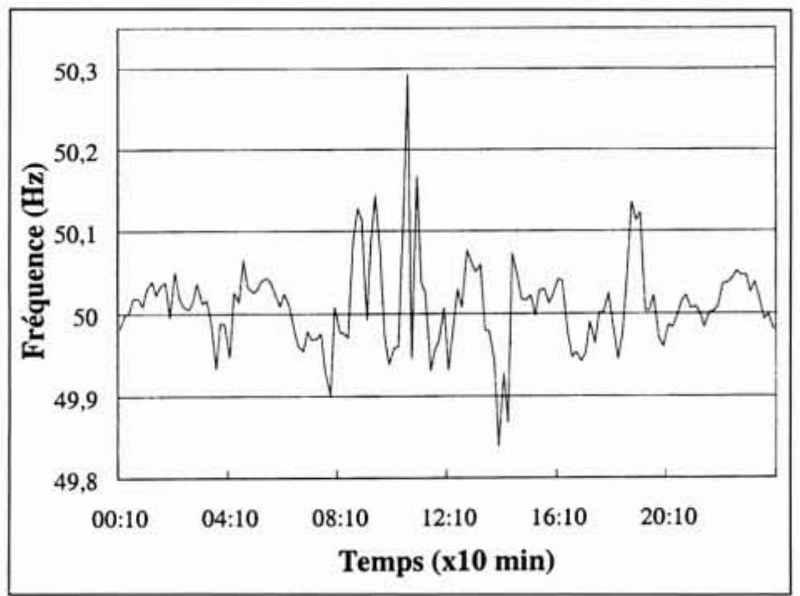

8. Mesures de fréquence au réseau. 

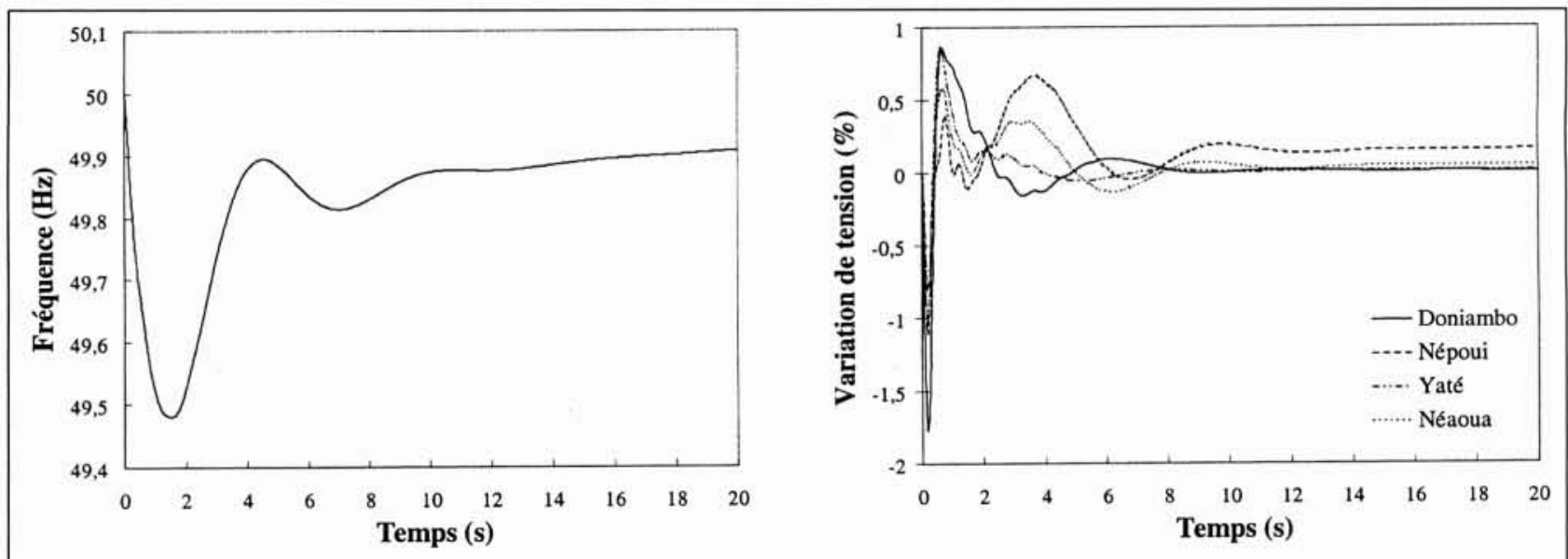

9. Variation de fréquence et de tension suite à une déconnexion d'une ferme éolienne de $20 \mathrm{MW}$.

court-circuit de la ligne connectant la ferme ou suite à une rafale de vent.

\section{CONCLUSIONS}

Dans cet article on a présenté des modèles de centrales hydrauliques et d'éoliennes connectées dans un système insulaire. Des modèles pour les unités conventionnelles sont présentés en [1$3]$. Le cas de la Nouvelle Calédonie, qui présente un grand intérêt grâce aux moyens divers de production, est examiné. Une autre particularité de ce cas est la forte demande industrielle qui a un impact important sur la stabilité transitoire du système électrique. Des résultats préliminaires ont montré que l'intégration d'une puissance éolienne importante n'affecte pas d'une façon significative le comportement dynamique du système. Dans une deuxième phase de cette étude, des simulations plus détaillées sont planifiées afin d'analyser des différentes types de perturbations plus complexes.

\section{REMERCIEMENTS}

L'auteur remercie la compagnie d'électricité ENERCAL en Nouvelle Calédonie et en particulier MM. J. Begaud, P. Nething et J. Nething pour leur précieuse collaboration pendant la mission effectuée sur site.

\section{RÉFÉRENCES}

[1] Stavrakakis G.S., Kariniotakis G.N. - « A general simulation algorithm for the detailed assessment of isolated Diesel-wind turbines interaction. Part I: A general multimachine power system model ». IEEE Transactions on Energy Conversion, Vol. 10, No. 5, Sept. 1995, pp. 577-583.

[2] Kariniotakis G.N., Stavrakakis G.S. - « A general simulation algorithm for the detailed assessment of isolated Diesel-wind turbines interaction. Part II : Implementation of the algorithm and case studies with induction generators $"$ IEEE Transactions on Energy Conversion, Vol. 10, No. 5, Sept. 1995, pp. 584-590.

[3] Kariniotakis G. - Contribution au développement d'un système de contrôle avancé pour les systèmes éolien-diesel. Thèse, Ecole des Mines de Paris, Centre d'Énergétique, Décembre 1996.

[4] Nogaret E., Stavrakakis G., Kariniotakis G. ET AL. - « An advanced control system for the optimal operation and management of medium size power systems with large penetration from renewable power sources ",.Renewable Energy, Elsevier Science Ltd., Vol. 12, 1997, pp. 137-149.

[5] IEEE Commitee report, « Dynamic models for steam and hydro turbines in power system studies $n$, IEEE PES Winter Meeting,New York, Jan. 28-Feb. 2, 1973. 